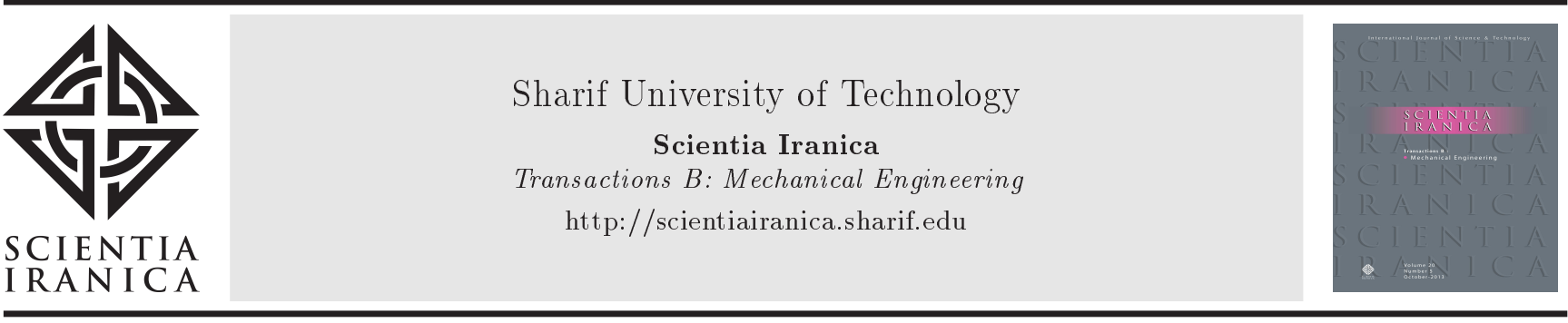

\title{
An optimal control strategy for parallel hybrid electric vehicles
}

\author{
M. Delkhosh and M. Saadat Foumani* \\ School of Mechanical Engineering, Sharif University of Technology, Tehran, P.O. Box 11155-9567, Iran.
}

Received 9 May 2016; received in revised form 24 February 2018; accepted 15 September 2018

\author{
KEYWORDS \\ Hybrid electric \\ vehicle; \\ Continuously variable \\ transmission; \\ Fuel consumption; \\ Emissions; \\ Speed ratio control; \\ Electric assist control \\ strategy; \\ Equivalent \\ consumption \\ minimization strategy.
}

\begin{abstract}
This paper proposes a new Power Management Strategy (PMS) for parallel hybrid electric vehicles equipped with Continuously Variable Transmission (CVT). The proposed PMS is established on the basis of Electric Assist Control Strategy (EACS) and Equivalent Consumption Minimization Strategy (ECMS). This control approach is based on maintaining the battery energy within a recommended range, considering the CVT efficiency in selecting the engine operating point, and finding the best power split between the engine and electric motor at certain moments of the driving. In order to evaluate the effectiveness of this scheme, it is compared with EACS, a modified version of EACS and ECMS. It is shown that, in all of the studied driving cycles, the proposed PMS is superior to the considered rival strategies in terms of fuel consumption and, also, HC and $\mathrm{CO}$ emissions.
\end{abstract}

(C) 2019 Sharif University of Technology. All rights reserved.

\section{Introduction}

Vehicles are one of the main energy consumers and important producers of airborne pollutants in the world. Hence, researchers have implemented many studies on the methods that reduce vehicles' Fuel Consumption (FC) and emissions. In this way, vehicle hybridization is recognized as one of the most promising technologies. In this technology, besides an internal combustion engine, another power source is employed to propel the vehicle [1]. One class of hybrid vehicles is Hybrid Electric Vehicle (HEV), in which an Electric Motor (EM) is used as the additional power source. The strategy of power split between HEV power sources is considered to be a major contributing

*. Corresponding author. Tel.: +982166165534 E-mail addresses: m_delkhosh@mech.sharif.ir (M. Delkhosh);m_saadat@sharif.ir(M. Saadat Foumani)

doi: $10.24200 /$ sci. 2018.20996 factor in the vehicle performance, its FC, and emissions levels. Power Management Strategies (PMSs) can be categorized into three types:

(i) The methods based on heuristic techniques, such as rule-based [2,3], and fuzzy logic $[4,5]$. One of the conventional heuristic methods is Electric Assist Control Strategy (EACS), which is currently employed in Toyota Prius [2] and Honda Insight [6];

(ii) The methods on the basis of instantaneous optimizations are of another type in which the electric energy consumption is converted to fuel consumption and an overall FC function is determined $[7,8]$. At each moment of the driving, this function is minimized and an optimal power split between the power sources is calculated. Equivalent Consumption Minimization Strategy (ECMS) is the most popular method of this group $[9,10]$;

(iii) The methods based on global optimizations are 
of the third type by which the global optimum of the controller is found. These methods can be developed on the basis of different global optimization tools, for instance, Dynamic Programming (DP) [11-13], Simulated Annealing (SA) [14,15], and Particle Swarm Optimization (PSO) $[16,17]$. It is notable that, due to the heavy calculation burden, the latter type cannot be used for realtime power management calculations. This type is only used to evaluate the effectiveness of the other strategies [9].

In the case of PMS optimization, some studies have been implemented. $\mathrm{Hu}$ et al. [18] proposed a convex programming method to minimize the level of $\mathrm{CO}_{2}$ in a plug-in hybrid electric vehicle. $\mathrm{Hu}$ et al. [19] employed a convex multi-criteria optimization method to find the optimal tradeoffs between the fuel-cell durability and hydrogen economy in the bus. Delkhosh et al. [20] optimized the powertrain and PMS in a parallel hybrid electric vehicle equipped with an infinitely variable transmission.

Besides the PMS, the power transmission is another factor that impacts the vehicle performance and, also, its FC and emissions. Recently, applications of Continuously Variable Transmissions (CVTs) as a power transmission system have become common for non-hybrid and hybrid vehicles, especially for Parallel HEVs (PHEVs) [21,22]. Contrary to a series HEV, in parallel one, an Internal Combustion Engine (ICE) is mechanically connected to the wheels and, therefore, cannot operate in optimal working regions unless a CVT-type transmission is used. The PMS of a PHEV equipped with CVT and the CVT speed ratio control are among the major concerns of researchers in this field $[2,4,23]$. As opposed to conventional transmissions, CVT's efficiency is highly sensitive to its operating condition [24,25]. Therefore, the PMS should take into account its efficiency in determining an optimum ICE operating point.

The previous studies on HEV control strategies demonstrate that the PMS has a significant impact on the vehicle performance, and a further investigation is still required in this area. The aim of this paper is to introduce a new PMS inspired by ECMS and a modified version of EACS (M-EACS, presented in [26]) to reach lower levels of $\mathrm{FC}$ and emissions. In this strategy, the power split is determined with regard to the battery State Of Charge (SOC). If SOC is below its recommended range, the ICE operating point is determined considering its optimal operating region and the CVT efficient working area. This method has been proposed in M-EACS. If SOC is within this range, an optimal power split between the power sources is determined using the instantaneous optimization method (which converts the electric energy usage to equivalent fuel consumption). This method is the same one used by ECMS to determine the optimal power split. This paper is structured as follows: First, the control method of a PHEV in a backward manner is described. Next, M-EACS and ECMS strategies are introduced briefly. Afterward, the proposed strategy is presented. Then, the considered baseline PHEV characteristics and the vehicle simulation model are explained. In order to investigate the proposed PMS, the FC, and emissions of the baseline vehicle in the case of using a devised strategy are compared with the cases of using EACS, M-EACS, and ECMS. Finally, the variations of the ICE and EM powers, the battery SOC, and the control parameters during a given driving cycle are exhibited.

\section{Power management strategy of PHEVs}

As stated before, the control strategy plays an important role in the performance of a given PHEV. The block diagram of the control system architecture for a PHEV equipped with CVT is shown in Figure 1.

The control system architecture exhibited in this figure employs a backward strategy. According to this diagram, the required power is calculated regarding the vehicle speed and acceleration obtained from the driving cycle. With regard to the battery energy level, the required power, and the vehicle speed, the control system defines the operating points of ICE and EM and, also, the speed ratio of CVT. It should be pointed out that, in the forward strategy, the required power is calculated according to the vehicle current speed and the driver's pedal displacement. The rest of the power management calculation process is similar to the backward type.

The proposed PMS in this study is a combination of ECMS and M-EACS. Hence, these techniques are described in the following.

\subsection{Modified EACS (M-EACS)}

EACS is one of the most popular PMS techniques among the existing rule-based control strategies for HEVs. According to this strategy, the ICE is the main power source, while EM is employed to propel the vehicle at low speeds and powers. This strategy attempts to maintain the battery SOC between its upper limit $\left(H_{S O C}\right)$ and lower limit $\left(L_{S O C}\right)$. This range is defined to prolong the battery service life and is presented by the battery manufacturer. The EACS strategy includes seven parameters to determine the operation points of ICE and EM [27]. The rules of this strategy are fully described in [20].

As discussed in [26], the EACS strategy has some shortcomings. The main shortcoming of EACS is the large number of its parameters, which increases its sensitivity to the driving behavior [16,28-30]. The op- 


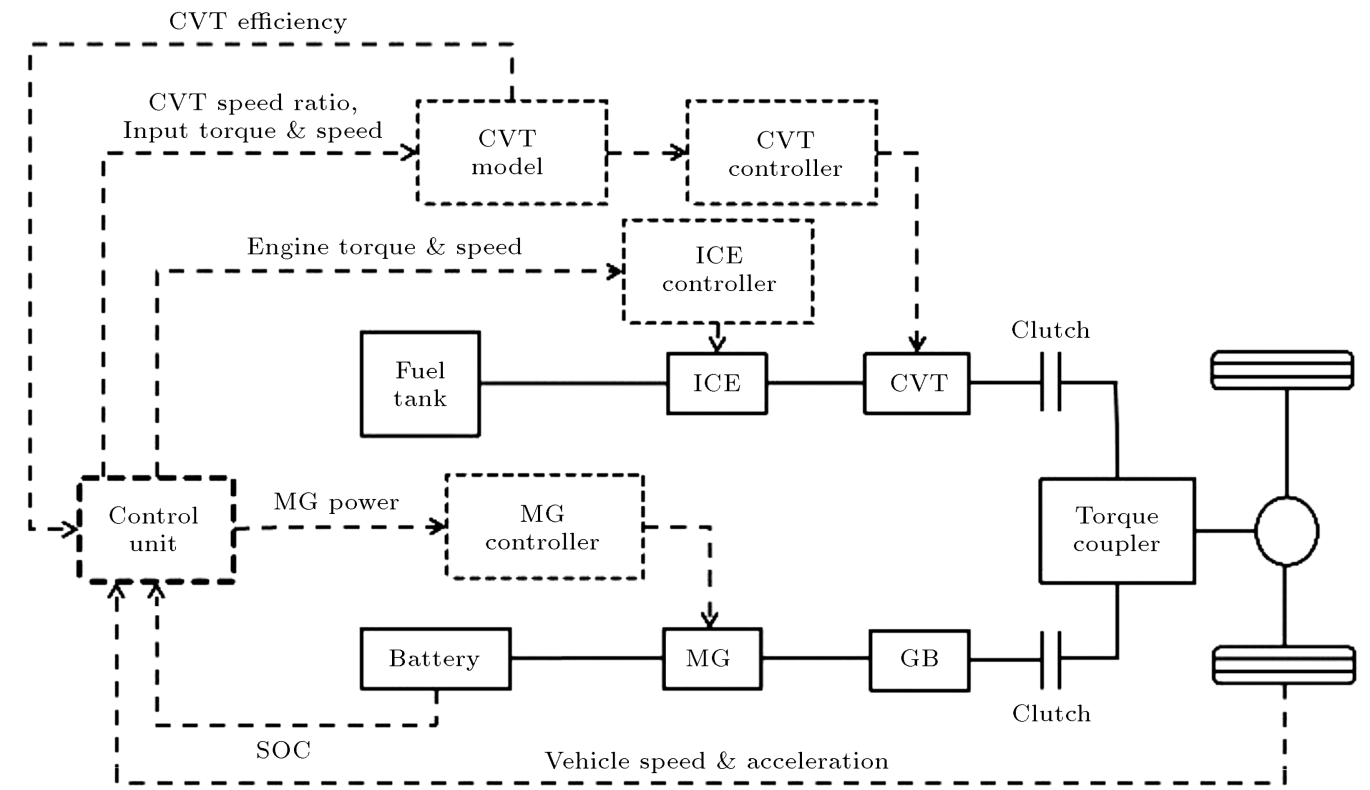

Figure 1. Block diagram of a PHEV's control system architecture.

timal values of these parameters should be determined by an optimization process for a predefined driving cycle. The optimization of different drive cycles may result in different optimal values and, therefore, the strategy optimized for a given drive cycle may be nonoptimal for another driving cycle. If a CVT is employed as the vehicle's transmission, there will be another shortcoming: the EACS does not take into account the transmission's efficiency in the process of defining the ICE's optimum operation point. The CVT's efficiency depends on its operating condition (contrary to the conventional transmission) [31-33]. Therefore, it is possible that, for the selected ICE operation point (by EACS), the CVT efficiency is low, which results in the poor overall efficiency of the vehicle's powertrain. Moreover, in this case, the operation point of ICE is determined regardless of the limitations on the speed ratio range of the transmissions.

The modifications proposed in [26] attempt to overcome these shortcomings. According to the modified strategy, at the moments when the ICE power is different from the required value (namely, the instants at which the ICE provides higher power that is used to propel the vehicle and charge the battery and the instants at which both ICE and EM provide the required power), the parameter that determines the operation point of ICE is the CVT speed ratio $\left(\tau_{C V T}\right)$. Based on this modification, the CVT speed ratio changes from its lowest value to the highest amount. The speed ratio for which the FC and emissions of the ICE are the lowest and also the CVT efficiency is the highest is identified as an optimal speed ratio. Then, the ICE rotational speed is determined according to the selected speed ratio and the vehicle speed. Finally, for the selected rotational speed, an optimal ICE power in terms of the
ICE's fuel consumption and emissions is found.

In summary, for M-EACS, the operation point of ICE is determined by the CVT speed ratio. As discussed in [26], three parameters of the EACS can be eliminated by applying the proposed method.

\subsection{Equivalent Consumption Minimization Strategy (ECMS)}

The ECMS strategy is on the basis of converting electric energy consumption to FC and minimizing the overall FC function. This function is calculated as follows:

$$
J(t, u)=\Delta E_{I C E}(t, u)+s(t) \Delta E_{E M}(t, u),
$$

where $\Delta E_{I C E}$ and $\Delta E_{E M}$ are the fuel and electric energy uses at the considered time interval, respectively. $u(t)$ is the control parameter, which is defined as follows: $T_{E M} / T_{r e q}$ (the EM torque divided by the required torque). In Eq. (1), $s(t)$ is the equivalence factor used to convert electric energy to fuel energy. This parameter is the key element of this strategy. At each moment of the driving, different values of $u(t)$ (which are within a predefined range) are considered, and some candidate operating points for the ICE are defined. For each candidate point, $s(t)$ is computed according to $u(t)$, the required energy at the wheels at this moment, the required energy (calculated from the driving beginning to this moment), and some fixed parameters $\left(s_{c h g}, s_{d i s}, \lambda\right)$ obtained from considering the vehicle in some predefined drive cycles. For calculating the equivalence factor for each candidate point, the cost function $(J(t, u))$ is computed, and the value of the control parameter for which $J(t, u)$ is minimal is considered as the optimal control parameter $\left(u_{\text {opt }}(t)\right)$. Therefore, an optimal power split between 
the power sources can be determined at each moment of driving [34].

ECMS has some downsides. For instance, its performance strongly depends on certain fixed parameters $\left(s_{\text {chg }}, s_{\text {dis }}, \lambda\right)$. These values are unknown a priori and, also, they are dependent on the considered driving cycle and may have different values in different drive cycles [34-36]. In addition, as discussed in [34-36], these parameters are the functions of the efficiencies of electrical and thermal paths in charge and discharge modes. If these efficiency values remain essentially unchanged for different driving schedules or the driving pattern is similar to the driving cycle considered in determining these parameters, their average over the considered drive cycles can be used in the ECMS strategy. If their values in various drive cycles are not close together, using their average may degrade the resulting performance. Another shortcoming of the ECMS is that it is a local optimization method. The optimal operation state of the HEV is determined regardless of the whole driving cycle, and the chosen state is a local optimal state. Therefore, the vehicle performance through this method may not be the best performance $[11,37]$.

\subsection{Proposed strategy}

In the proposed PMS, three cases regarding the battery SOC are considered for the vehicle:

1. $S O C>H_{S O C}$. In order to prolong the battery service life, it is necessary to maintain its energy level in the given recommended range. For this purpose, in this case, the EM should propel the vehicle until the battery SOC falls within the recommended range. If the required power exceeds the maximum electric power, which can be transferred (limited by the battery maximum discharge rate and the maximum EM power), the EM provides maximum allowable power and the ICE provides the rest of the required power. If the vehicle is in the braking mode, the battery is not charged and the mechanical brakes dissipate the vehicle kinetic energy;

2. $L_{S O C}<S O C<H_{S O C}$. In this case, different modes can be applied: pure thermal, pure electric, and charge and discharge modes. In the charge mode, the ICE provides a power more than the demanded value, and additional power will be used to charge the battery. Similarly, in the discharge mode, the EM is used to propel the vehicle. Obviously, in order to compare different modes and select the optimum one, it is necessary to convert the electric energy to the equivalent fuel energy. This aim is achievable using the technique employed by the ECMS strategy. This method was presented in the previous section. At any moment of driving, tentative values of the control parameter $u(t)$ in its allowable range are applied, and the values of function $J(t)$ are computed; as a result, an optimal control parameter $\left(u_{\text {opt }}(t)\right)$ is found. By determining $u_{\text {opt }}(t)$, the optimum driving mode can be defined, and the values of ICE and EM powers can be found.

It should be noted that if the vehicle is in the braking mode, the braking energy is stored in the battery by the EM, which functions as a generator.

Obviously, the goal of the PMS (for instance, reducing the $\mathrm{FC}$, emissions, or both) should be considered in formulating the cost function. In the present study, the aim of the proposed PMS is to minimize the vehicle $\mathrm{FC}$ and emissions, simultaneously.

In some studies, besides electric energy consumption, the FC alone is considered in the definition of cost function [34,38]. In some other studies, in addition to the FC, emissions are considered in the formulation of this function using a weighted summation method $[35,36]$. This method is used to convert different objectives into a single scalar function. As demonstrated in several pieces of literature [39-41], the weighted sum method is sometimes unable to reach the global minimum point, especially for non-convex spaces. Another method for this aim is Global Criterion method (GC). In this method, first, the minimum of each objective term is calculated without considering other terms. Then, a scalar objective function is defined as the sum of the normalized Euclidean distances between each objective and its minimum value [42]. This method does not have the disadvantages of weighted sum method and is able to reach the Pareto front [43].

Due to the above-mentioned reasons, the cost function is defined using the GC method as given below:

$$
\begin{aligned}
J_{G C}(t, u)=\{ & w_{1}\left(\frac{J(t, u)-J_{\min }(t)}{J_{\min }(t)}\right)^{2} \\
& +w_{2}\left(\frac{\mathrm{CO}(t, u)-\mathrm{CO}_{\min }(t)}{\mathrm{CO}_{\min }(t)}\right)^{2} \\
& +w_{3}\left(\frac{\mathrm{HC}(t, u)-\mathrm{HC}_{\min }(t)}{\mathrm{HC}_{\min }(t)}\right)^{2} \\
& \left.+w_{4}\left(\frac{\mathrm{NOx}(t, u)-\mathrm{NOx}_{\min }(t)}{\mathrm{NOx}_{\min }(t)}\right)^{2}\right\},
\end{aligned}
$$

where $\mathrm{NOx}, \mathrm{CO}$, and $\mathrm{HC}$ denote different pollutant emissions in $\mathrm{gr} / \mathrm{kWh}$, and the 'min' index means the minimum of each function regardless of the other objectives. Moreover, $w_{i}$ means the relative importance of the ith function. In this study, the 
FC and emissions are of the same importance. Therefore, the related weights have been considered to be equal. By minimizing the function $J_{G C}$ at each moment of driving, an optimum control parameter, which results in the lowest levels of FC and emissions, can be found;

3. $S O C<L_{S O C}$. In this case, since the battery energy is below the recommended range, it is necessary to charge the battery by the braking energy or ICE. If the vehicle is in the braking mode, similar to the previous case, the battery is charged by the EM. Otherwise, the ICE will provide both the required power and charge power. For this case, the M-EACS approach presented in the previous section is used.

According to the mentioned rules, the proposed PMS has fewer parameters than that of EACS. Therefore, its sensitivity to the driving cycle will be lower than that of EACS. Furthermore, in contrast to EACS, the transmission efficiency is taken into account in determining the ICE operation point. On the other hand, the proposed PMS uses an instantaneous optimization to find the ICE and EM working points only in certain conditions. As discussed in the previous section, ECMS has some predefined parameters $\left(s_{c h g}\right.$, $\left.s_{d i s}, \lambda\right)$, which are the functions of the driving pattern and the efficiency of thermal and electrical paths. Since the proposed PMS uses the ECMS approach partially, its sensitivity to the driving cycle will be lower than that of ECMS.

\section{Application of the proposed PMS}

In this section, the proposed PMS is used to control a baseline PHEV and, also, the results are compared with those of other strategies.

\subsection{Vehicle characteristics}

The control system architecture of the baseline vehicle considered in this study is the same as that shown in Figure 1. The characteristics of the vehicle components are also presented in Table 1.

In order to simulate the vehicle behavior in different conditions, the model presented in [26] is used. This model was developed in MATLAB for the baseline vehicle when it is equipped with CVT. The vehicle simulation model includes a look-up table to represent the EM efficiency, a model of full-toroidal CVT, the battery efficiency data, and some empirical maps for the ICE's FC and emissions, as well as the used powersplit control strategy. In [26], this model has been verified by comparing its simulation results with some experimental data.

\subsection{Evaluating the proposed PMS}

In order to evaluate the performance of the proposed PMS, it should be compared with other strategies. In this section, this strategy is compared with the EACS, M-EACS, and ECMS strategies. As discussed before, EACS and M-EACS techniques have some parameters that impact their performances. Hence, the parameters of these methods should be firstly optimized to find the optimum PMSs before comparing them with the proposed PMS. In order to implement the optimization, Particle Swarm Optimization (PSO) is used with the aim of minimizing the function introduced in Eq. (3):

$$
\begin{gathered}
F=\left\{w_{1}\left(\frac{F C-F C_{\min }}{F C_{\min }}\right)^{2}+w_{2}\left(\frac{\mathrm{CO}-\mathrm{CO}_{\min }}{\mathrm{CO}_{\min }}\right)^{2}\right. \\
+w_{3}\left(\frac{\mathrm{HC}-\mathrm{HC}_{\min }}{\mathrm{HC}_{\min }}\right)^{2} \\
\left.+w_{4}\left(\frac{\mathrm{NOx}^{2} \mathrm{NOx}_{\min }}{\mathrm{NOx}_{\min }}\right)^{2}\right\}^{\frac{1}{2}}
\end{gathered}
$$

As can be seen, the GC technique is employed to define the objective function, similar to the previous section. Minimizing this function leads to an effective PMS in terms of emissions and FC criteria. In this function, FC is the vehicle fuel consumption $(L / 100 \mathrm{~km})$ for a given drive cycle. Moreover, $\mathrm{CO}, \mathrm{HC}$, and NOx are emissions in $\mathrm{gr} / \mathrm{km}$. Similar to the previous section, the 'min' index denotes the minimal value of each objective term obtained regardless of the others.

In the case of running ECMS, it is necessary to assign an average value to its constant parameters $\left(s_{c h g}, s_{d i s}, \lambda\right)$. To this end, different driving cycles are considered and these parameters are calculated [34]. Then, their averages over the considered driving cycles are regarded as their optimal values and, then, used in the ECMS method.

The considered driving cycle to compare the above strategies is SC03. The reason for selecting this cycle is its aggressive accelerations and decelerations, meaning that the hybridization aspect can be evaluated accurately.

Table 2 shows the results of using the proposed PMS, optimized EACS, optimized M-EACS, and ECMS as the controllers of the considered vehicle in the SC03 drive cycle.

According to Table 2, for this driving cycle, the levels of $\mathrm{FC}, \mathrm{CO}$, and $\mathrm{HC}$ in the case of employing the proposed PMS are lower than those in the cases of employing the optimized EACS, optimized M-EACS, and also ECMS. The NOx levels for the proposed PMS and the optimized M-EACS are approximately equal, while these values are more than the those corresponding to the optimized EACS case. This is 
Table 1. Specifications of the HEV subsystems.

\begin{tabular}{|c|c|}
\hline Element & Characteristics \\
\hline \multicolumn{2}{|l|}{ Internal combustion engine [44] } \\
\hline Volume & $1.3 \mathrm{~L}$ \\
\hline Maximum power & $53.2 \mathrm{~kW}$ at $5200 \mathrm{rpm}$ \\
\hline Maximum torque & $113 \mathrm{Nm}$ at $2800 \mathrm{rpm}$ \\
\hline Peak efficiency & 0.34 \\
\hline Electric motor/generator [45] & Asynchronous induction motor/generator \\
\hline Maximum power & $30 \mathrm{~kW}$ \\
\hline Maximum torque & $300 \mathrm{Nm}$ \\
\hline Maximum speed & $6000 \mathrm{rpm}$ \\
\hline Peak efficiency & 0.9 \\
\hline Minimum voltage & $60 \mathrm{~V}$ \\
\hline Battery [46] & Lithium-ion polymer rechargeable \\
\hline Number of modules & 96 \\
\hline Nominal capacity & $10.05 \mathrm{Ah}$ \\
\hline Nominal voltage & $14.8 \mathrm{~V}$ \\
\hline Internal impedance & $15 \mathrm{~m} \Omega$ \\
\hline Maximum allowable current & 10.05 A (charge), 120 A (discharge) \\
\hline Vehicle [44] & Light passenger car \\
\hline Frontal area & $1.94 \mathrm{~m}^{2}$ \\
\hline Rolling resistance & 0.014 \\
\hline Drag coefficient & 0.46 \\
\hline Wheel radius & $0.264 \mathrm{~m}$ \\
\hline Cargo mass & $136 \mathrm{~kg}$ \\
\hline Total mass & $1224 \mathrm{~kg}$ \\
\hline Power train $[47]$ & Continuously variable transmission \\
\hline Efficiency & Variable with respect to input torque, speed and speed ratio \\
\hline Differential speed ratio and efficiency & $3.778,97 \%$ \\
\hline Torque coupler & One-speed gear mate \\
\hline
\end{tabular}

Table 2. FC and emissions during SC03 cycle for different control strategies.

\begin{tabular}{lcccc}
\hline Control strategy & FC $(\mathbf{L} / \mathbf{1 0 0} \mathbf{~ k m})$ & CO $(\mathbf{g r} / \mathbf{k m})$ & HC $(\mathbf{g r} / \mathbf{k m})$ & NOx $(\mathbf{g r} / \mathbf{k m})$ \\
\hline Proposed PMS & 4.25 & 3.29 & 0.66 & 1.39 \\
Optimized M-EACS & 4.33 & 3.44 & 0.66 & 1.38 \\
Optimized EACS & 4.48 & 3.80 & 0.70 & 1.25 \\
ECMS & 4.54 & 3.91 & 0.74 & 1.42 \\
\hline
\end{tabular}

because, in the ICE emissions map, the NOx-optimum operating points are in the high power area (Figure 2), where the CVT efficiency is a low value. Therefore, by applying the proposed PMS, the ICE will operate at lower powers where the CVT unit is efficient and, thus, the NOx emission is at a high level. In order to decrease the NOx level of ICE in the case of using the proposed PMS, one solution is to increase the importance weight of $\mathrm{NOx}\left(w_{4}\right)$ in the formulations of $J_{G C}$ function (Eq. (2)). For example, if $w_{4}=0.5$ and $w_{1}=w_{2}=w_{3}=0.167$, the vehicle $\mathrm{FC}$ and emissions in the case of using the proposed PMS will be as follows: $\mathrm{FC}=4.38 \mathrm{~L} / 100 \mathrm{~km}, \mathrm{CO}=3.65 \mathrm{gr} / \mathrm{km}$, $\mathrm{HC}=0.70 \mathrm{gr} / \mathrm{km}$, and $\mathrm{NOx}=1.24 \mathrm{gr} / \mathrm{km}$. As can be seen, by this approach, the NOx level decreases, while the values of other functions will increase. 


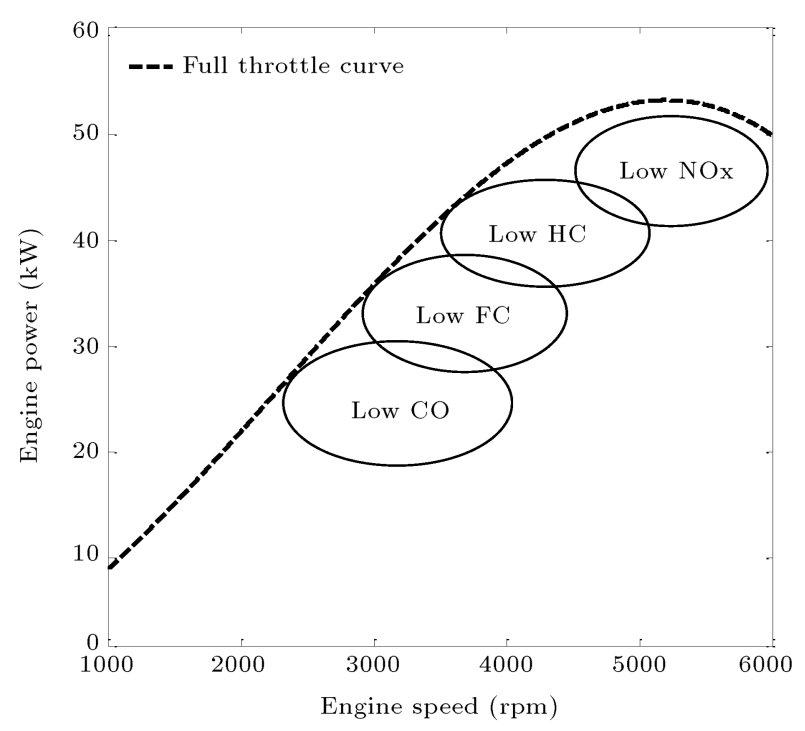

Figure 2. Optimal regions of the vehicle ICE in terms of FC and pollutant emissions [20].

In order to accurately compare the proposed PMS with EACS, M-EACS, and ECMS techniques, these strategies should be evaluated in other driving cycles, too. Hence, Urban Driving Cycle (ECE), Urban Dynamometer Driving Schedule (UDDS), IM240, and Federal Test Procedure (FTP) cycles are also considered. The vehicle FC and emissions in these cycles in the case of using each of the mentioned strategies are shown in Figure 3. In this figure, the results are normalized with respect to the proposed PMS case. As can be seen, for these driving cycles, the results are the same as before.
Figure 4 shows the variations of the vehicle speed, ICE and EM powers, battery SOC, control parameter $(u(t))$, and $\tau_{C V T}$ in the first 70 seconds of the SC03 cycle for the proposed PMS.

The figure reveals that, during the braking, the engine is turned off, the CVT speed ratio is unchanged, and the battery SOC increases. Furthermore, all of the required power (braking power) is supplied by the EM. At these moments, neither $u(t)$ nor the CVT speed ratio is the control parameter. Furthermore, while the vehicle is at the standstill, the ICE and EM are turned off, the battery SOC and the CVT speed ratio are fixed, and $u(t)=0$. In the first 43 seconds of the considered driving cycle, the battery SOC is lower than $L_{S O C}$. According to the rules of the proposed PMS, at these times (except for the braking and stop times), the control parameter is the CVT speed ratio and $u(t)=0$. In the rest of the considered simulation time, since $S O C>L_{S O C}, u(t)$ is the control parameter and, also, the CVT speed ratio is determined according to the operating point of ICE, which is determined with regard to $u(t)$. According to the figure, while $u(t)<0$, the EM is in the generator mode (negative power). At these moments, the ICE provides a power amount more than the required power in order to charge the battery by the generator. Moreover, as can be observed, while $u(t)>0$, the EM is in the motor mode. At these moments, both ICE and EM propel the vehicle (discharge mode). Therefore, the battery SOC decreases at these times.

For a major portion of the moments when $u(t)$ is the control parameter, the absolute value of $u(t)$ is

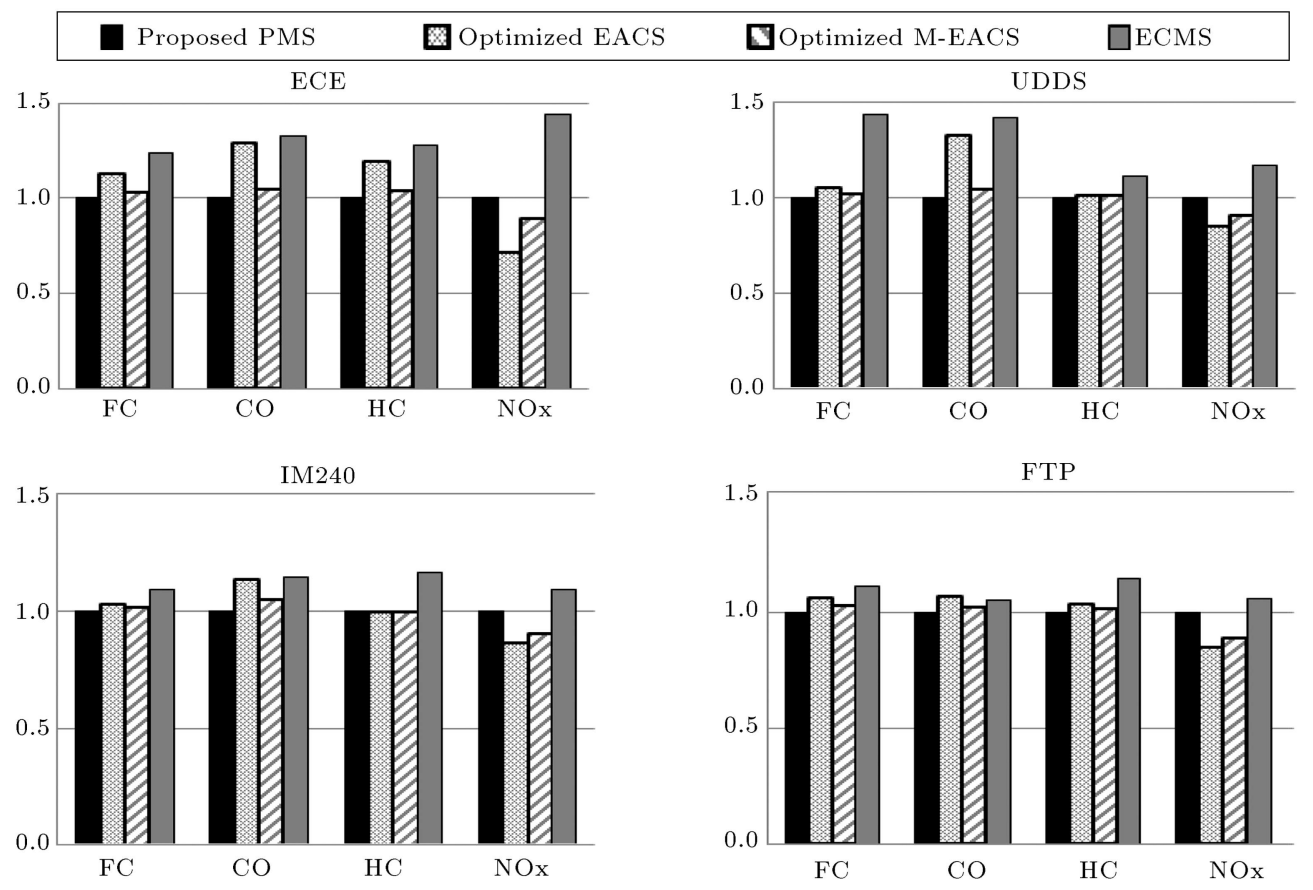

Figure 3. Normalized FC and emissions in the other considered driving cycles. 

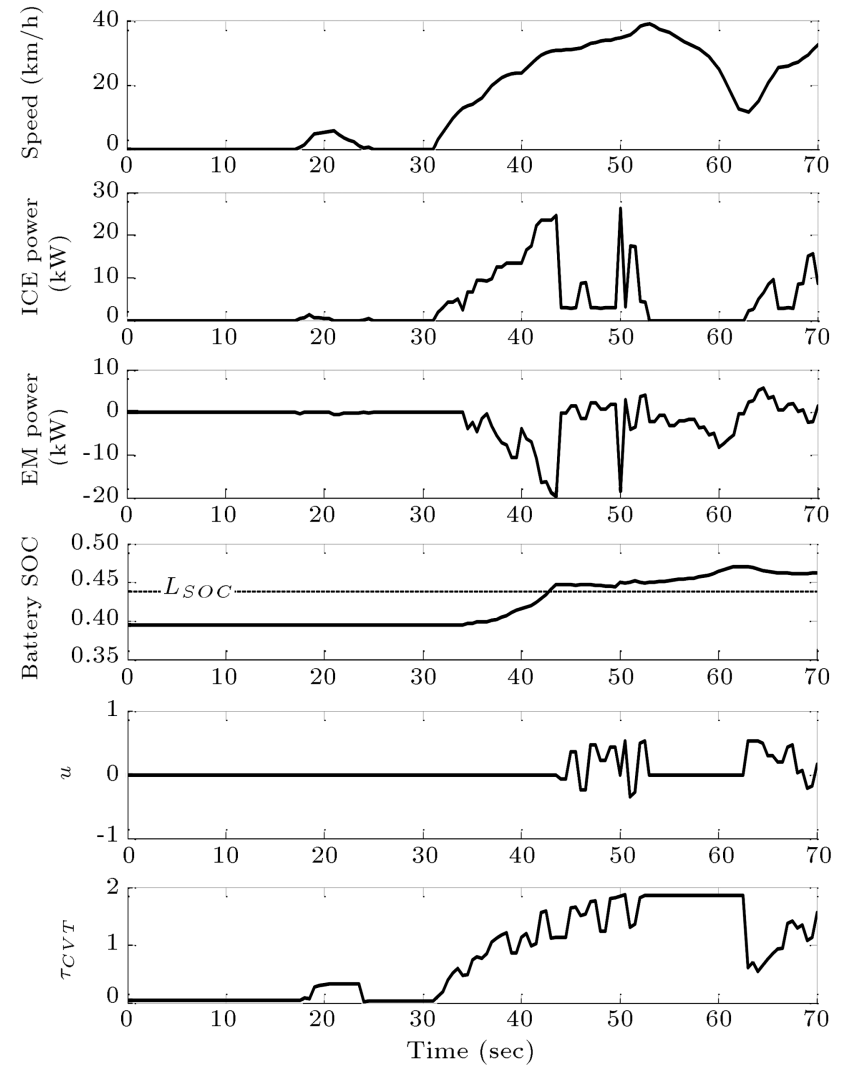

Figure 4. Simulation results with the proposed PMS for the first 70 seconds of the SC03 cycle.

lower than 0.5, which means that the ICE power is more than the absolute value of the EM power. This is because the ICE is inefficient at the low powers and, therefore, it is reasonable to use it at the high powers (see Figure 2).

\section{Conclusion}

In this study, a new PMS for PHEVs equipped with CVT was presented and, also, its effectiveness was demonstrated by some simulation results. Based on this strategy, in some conditions, the best working point of the ICE was determined by changing the CVT speed ratio and, also, the battery was charged by the ICE. Moreover, in some occasions, a control parameter was used to determine the best power distribution between the ICE and EM. For a given baseline PHEV, the comparisons between the proposed PMS, the ECMS, and the optimized versions of EACS and M-EACS revealed that, in all of the considered driving cycles, the proposed PMS was superior to the other rival strategies in terms of $\mathrm{FC}, \mathrm{HC}$, and $\mathrm{CO}$. Further, it was shown that the vehicle NOx in the case of using the proposed PMS was more than that of the EACS and M-EACS techniques. For example, the reduction percentage of $\mathrm{FC}, \mathrm{CO}$, and $\mathrm{HC}$ with respect to the optimized EACS in the SC03 drive cycle included $5.4 \%, 15.5 \%$, and $6.1 \%$, respectively, while the NOx growth was $10.1 \%$. As shown earlier, if the importance weight of $\mathrm{NOx}$ in the formulations of cost functions $\left(J_{G C}\right.$ (Eq. (2))) increased, the level of NOx would decrease, while the amounts of FC and the other emissions would increase. Hence, the best importance weight of NOx should be determined according to the designer goals.

\section{Nomenclature}

$\begin{array}{ll}J & \text { Cost function } \\ H_{S O C} & \text { Upper limit of battery recommended } \\ & \text { SOC } \\ L_{S O C} & \text { Lower limit of battery recommended } \\ & \text { SOC } \\ P_{\text {req }} & \text { Required power } \\ P_{I C E \text { max }} & \text { ICE maximum power } \\ s(t) & \text { Equivalence factor } \\ u & \text { Control parameter } \\ w & \text { Importance weight } \\ V_{L} & \text { Lunch speed } \\ \Delta E_{I C E} & \text { Fuel energy use } \\ \Delta E_{E M} & \text { Electric energy use } \\ \tau_{C V T} & \text { CVT speed ratio } \\ \eta_{C V T} & \text { CVT efficiency }\end{array}$

\section{References}

1. Martinez, C.M., Hu, X., Cao, D., Velenis, E., Gao, B., and Wellers, M. "Energy management in plugin hybrid electric vehicles: recent progress and a connected vehicles perspective", IEEE Trans. Veh. Technol., 66(6), pp. 4534-4549 (2017).

2. Banvait, H., Anwar, S., and Chen, Y., "A rulebased energy management strategy for Plug-in Hybrid Electric Vehicle (PHEV)", ACC '09., pp. 3938-3943 (2009).

3. Boukehili, A., Zhang, Y., and Sun, S. "Simulation and comparison of HEV battery control for best fuel economy and longer battery life", WEVJ, 4, pp. 421426 (2010).

4. Dorri, M. and Shamekhi, A.H. "Design and optimization of a new control strategy in a parallel hybrid electric vehicle in order to improve fuel economy", $P I$ Mech. Eng. D-J Aut., 225(6), pp. 747-759 (2011).

5. Majdi, L., Ghaffari, A., and Fatehi, N. "Control strategy in hybrid electric vehicle using fuzzy logic controller", Robio, pp. 842-847 (2009).

6. Safaei, A., Ha'iri-Yazdi, M.R., Esfahanian, V., Esfahanian, M., Tehrani, M.M., and Nehzati, H. "Designing an intelligent control strategy for hybrid powertrains utilizing a fuzzy driving cycle identification agent", $P$ I Mech. Eng. D-J Aut., 229(9), pp. 1169-1188 (2014). 
7. Pisu, P. and Rizzoni, G. "A supervisory control strategy for series hybrid electric vehicles with two energy storage systems", VPPC, pp. 65-72 (2005).

8. Park, J. and Park, J.-H. "Development of equivalent fuel consumption minimization strategy for hybrid electric vehicles", Int. J. of Automot Techn., 13(5), pp. 835-843 (2012).

9. Wang, F., Mao, X.-J., Zhuo, B., Zhong, H., and Ma, Z.-L. "Parallel hybrid electric system energy optimization control with automated mechanical transmission", P I Mech. Eng. D-J AUT., 223(2), pp. 151-167 (2009).

10. Sinoquet, D., Rousseau, G., and Milhau, Y. "Design optimization and optimal control for hybrid vehicles", Optim. Eng., 12(1), pp. 199-213 (2009).

11. Mansour, C. and Clodic, D. "Optimized energy management control for the Toyota hybrid system using dynamic programming on a predicted route with short computation time", Int. J. of Automot. Techn., 13(2), pp. 309-324 (2012).

12. Guemri, M., Neffati, A., Caux, S., and Ngueveu, S.U. "Management of distributed power in hybrid vehicles based on D.P. or fuzzy logic", Optim. Eng., 15(4), pp. 993-1012 (2013).

13. Grothey, A. and Yang, X. "Top-percentile traffic routing problem by dynamic programming", Optim. Eng., 12(4), pp. 631-655 (2011).

14. Gao, W. and Porandla, S.K. "Design optimization of a parallel hybrid electric powertrain", $V P P C$, pp. 530$535(2005)$.

15. Wang, Z., Huang, B., Xu, Y., and Li, W. "Optimization of series hybrid electric vehicle operational parameters by simulated annealing algorithm", ICC, pp. 1536-1541 (2007).

16. Wu, J., Zhang, C.-H., and Cui, N.-X. "PSO algorithmbased parameter optimization for HEV powertrain and its control strategy", Int. J. of Automot. Techn., 9(1), pp. 53-59 (2008).

17. Wu, X., Cao, B., Wen, J., and Bian, Y. "Particle swarm optimization for plug-in hybrid electric vehicle control strategy parameter", VPPC, pp. 1-5 (2008).

18. Hu, X., Moura, S.J., Murgovski, N., Egardt, B., and Cao, D. "Integrated optimization of battery sizing, charging, and power management in plug-in hybrid electric vehicles", IEEE T Contr. Syst. T, 24(3), pp. 1036-1043 (2016).

19. Hu, X., Jiang, J., Egardt, B., and Cao, D. "Advanced power-source integration in hybrid electric vehicles: Multicriteria optimization approach", IEEE Trans. Ind. Electron., 62(12), pp. 7847-7858 (2015).

20. Delkhosh, M., Saadat Foumani, M., and Rostami, P. "Optimization of powertrain and control strategy of hybrid electric vehicle", Sci. Iran, 22(5), pp. 1842-1854 (2015).
21. Ryu, W., Cho, N., Yoo, I., Song, H., and Kim, H. "Performance analysis of a CVT clutch system for a hybrid electric vehicle", Int. J. of Automot. Techn., 10(1), pp. 115-121 (2009).

22. Wang, C.-L., Yin, C.-L., Zhang, T., and Zhu, L. "Powertrain design and experiment research of a parallel hybrid electric vehicle", Int. J. of Automot. Techn., 10(5), pp. 589-596 (2009).

23. Suh, B., Chang, Y.H., Han, S.B., and Chung, Y.J. "Simulation of a powertrain system for the diesel hybrid electric bus", Int. J. of Automot. Techn., 13(5), pp. 701-711 (2012).

24. Hu, X., Wang, H., and Tang, X. "Cyber-physical control for energy-saving vehicle following with connectivity", IEEE Trans. Ind. Electron., 64(11), pp. 85788587 (2017).

25. Carbone, G., Mangialardi, L., and Mantriota, G. "A comparison of the performances of full and half toroidal traction drives", Mech. Mach. Theory, 39(9), pp. 921942 (2004).

26. Delkhosh, M., Saadat Foumani, M., Azad, N.L., and Rostami, P. "A new control strategy for hybrid electric vehicles equipped with continuously variable transmission", P I Mech. Eng. D-J Aut., 230(6), pp. 803-816 (2015)

27. Delkhosh, M., Saadat Foumani, M., and Falahati, F. "A modified control strategy for parallel hybrid electric vehicles equipped with continuously variable transmission", Sci. Iran., 23(3), pp. 966-975 (2016).

28. Montazeri-Gh, M., Poursamad, A., and Ghalichi, B. "Application of genetic algorithm for optimization of control strategy in parallel hybrid electric vehicles", $J$. of Franklin Inst., 343(4), pp. 420-435 (2006).

29. Montazeri-Gh, M. and Poursamad, A. "Application of genetic algorithm for simultaneous optimisation of HEV component sizing and control strategy", Int. J. Altern. Propul., 1(1), pp. 63-78 (2006).

30. Long, V.T. and Nhan, N.V. "Bees-algorithm-based optimization of component size and control strategy parameters for parallel hybrid electric vehicles", Int. J. of Automot. Techn., 13(7), pp. 1177-1183 (2012).

31. Delkhosh, M., Saadat Foumani, M., Boroushaki, M., Ekhtiari, M., and Dehghani, M. "Geometrical optimization of half toroidal continuously variable transmission using particle swarm optimization", Sci. Iran., 18(5), pp. 1126-1132 (2011).

32. Delkhosh, M., Saadat Foumani, M., and Boroushaki, M. "Geometrical optimization of parallel infinitely variable transmission to decrease vehicle fuel consumption", Mech. Based Des Struc., 24(4), pp. 483-501 (2014).

33. Delkhosh, M. and Saadat Foumani, M. "Optimisation of full-toroidal continuously variable transmission in conjunction with fixed ratio mechanism using particle swarm optimisation", Vehicle Syst. Dyn., 51(5), pp. 671-683 (2013). 
34. Sciarretta, A., Back, M., and Guzzella, L. "Optimal control of parallel hybrid electric vehicles", IEEE T Contr. Syst. T, 12(3), pp. 352-363 (2004).

35. Musardo, C., Staccia, B., Midlam-Mohler, S., Guezennec, Y., and Rizzoni, G. "Supervisory control for NOx reduction of an HEV with a mixed-mode HCCI/CIDI engine", $A C C$, pp. 3877-3881 (2005).

36. Pisu, P. and Rizzoni, G. "A comparative study of supervisory control strategies for hybrid electric vehicles", IEEE T Contr. Syst. T, 15(3), pp. 506-518 (2007).

37. Fan, B.S.-M., Multidisciplinary Optimization of Hybrid Electric Vehicles: Component Sizing and Power Management Logic, University of Waterloo (2011).

38. Delprat, S., Guerra, T.-M., and Rimaux, J. "Optimal control of a parallel powertrain: from global optimization to real time control strategy", IEEE Veh. Technol. Conf., 4, pp. 2082-2088 (2002).

39. Statnikov, R.B. and Matusov, J.B., Multicriteria Optimization and Engineering, 1st Ed., Springer (1995).

40. Marler, R.T. and Arora, J.S. "Survey of multiobjective optimization methods for engineering", Struct Multidisc. Optim., 26(6), pp. 369-395 (2004).

41. Das, I. and Dennis, J.E. "A closer look at drawbacks of minimizing weighted sums of objectives for Pareto set generation in multicriteria optimization problems", Struct. Optim., 14(1), pp. 63-69 (1997).

42. Marler, R.T. and Arora, J.S. "Survey of multiobjective optimization methods for engineering", Struct. Multidisc. Optim., 26(6), pp. 369-395 (2004).
43. Yu, P.L. "A class of solutions for group decision problems", Management Sci., 19(8), pp. 936-946 (1973).

44. "Saipa corporation", [Online]. Available: http://www. saipacorp.com/portal/Home/.

45. "ADVISOR library reorganized structure", http:// adv-vehiclesim.sourceforge.net/LibReorg.html (2003).

46. "Gita battery", [Online]. Available: http://www. gitabattery.com/.

47. Delkhosh, M. and Saadat Foumani, M. "Multiobjective geometrical optimization of full toroidal CVT", Int. J. of Automot. Techn., 14(5), pp. 707-715 (2013).

\section{Biographies}

Mojtaba Delkhosh received his BSc, MSc, and PhD degrees in Mechanical Engineering from Sharif University of Technology, Tehran, Iran in 2009, 2011, and 2016, respectively. Currently, he is the post-doctoral researcher in Sharif University of Technology. His fields of interests are vehicle dynamics, design of hybrid vehicles, machine design, vibration analysis, power transmission systems, and optimization algorithms.

Mahmoud Saadat Foumani received his $\mathrm{PhD}$ degree in Mechanical Engineering from Sharif University of Technology, Tehran, Iran in 2002. He was a Faculty member at Semnan University from 2002 to 2006 and is now a faculty member of Sharif University of Technology, Mechanical Engineering Department. He teaches courses at the 'Applied Design group' at undergraduate and graduate levels. His teaching focuses on mechanical engineering design, vehicle dynamics, chassis design, and advanced mathematics. 